\title{
Ventricular arrhythmia in heart failure patients with reduced ejection fraction and central sleep apnoea
}

\author{
Christoph Fisser $\mathbb{D}^{1}$, Jannis Bureck ${ }^{1}$, Lara Gall ${ }^{1}$, Victoria Vaas ${ }^{1}$, Jörg Priefert ${ }^{1}$, Sabine Fredersdorf ${ }^{1}$, \\ Florian Zeman ${ }^{2}$, Dominik Linz ${ }^{3,4,5,6}$, Holger Wöhrle ${ }^{7}$, Renaud Tamisier $\mathbb{C}^{8}$, Helmut Teschler ${ }^{9}$, \\ Martin R. Cowie ${ }^{10}$ and Michael Arzt ${ }^{1}$
}

\begin{abstract}
${ }^{1}$ Dept of Internal Medicine II, University Medical Centre Regensburg, Regensburg, Germany. ${ }^{2}$ Center for Clinical Studies, University Hospital Regensburg, Regensburg, Germany. ${ }^{3}$ Dept of Cardiology, Maastricht University Medical Centre and Cardiovascular Research Institute Maastricht, Maastricht, The Netherlands. ${ }^{4}$ Dept of Cardiology, Radboud University Medical Centre, Nijmegen, the Netherlands. ${ }^{5}$ Dept of Biomedical Sciences, Faculty of Health and Medical Sciences, University of Copenhagen, Copenhagen, Denmark. ${ }^{6}$ Centre for Heart Rhythm Disorders, Royal Adelaide Hospital, University of Adelaide, Adelaide, Australia. ${ }^{7}$ Sleep and Ventilation Center Blaubeuren, Lung Center Ulm, Ulm, Germany. ${ }^{8} \mathrm{HP} 2$ Laboratory, INSERM U1042, Grenoble Alpes University, Sleep laboratory, Pole Thorax et Vaisseaux, Grenoble Alps University Hospital, Grenoble, France. ${ }^{9}$ Dept of Pneumology, AFPR, Ruhrlandklinik, West German Lung Center, University Medicine Essen, Essen, Germany. ${ }^{10}$ Royal Brompton Hospital \& School of Cardiovascular Medicine \& Sciences, Faculty of Life Sciences \& Medicine, King's College London, London, UK.
\end{abstract}

Corresponding author: Christoph Fisser (christoph.fisser@ukr.de)

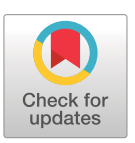

Copyright $\odot$ The authors 2021

This version is distributed under the terms of the Creative Commons Attribution NonCommercial Licence 4.0. For commercial reproduction rights and permissions contact permissions@ersnet.org

This article has supplementary material available from openres.ersjournals.com

Received: 5 March 2021 Accepted: 18 May 2021

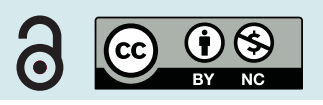

Shareable abstract (@ERSpublications)

High ventricular arrhythmia burden occurs in $44 \%$ of heart failure patients with reduced ejection fraction and central sleep apnoea. Arrhythmia burden is higher in sleep phases with versus without Cheyne-Stokes respiration. https://bit.ly/2QBDamC

Cite this article as: Fisser C, Bureck J, Gall L, et al. Ventricular arrhythmia in heart failure patients with reduced ejection fraction and central sleep apnoea. ERJ Open Res 2021; 7: 00147-2021 [DOI: 10.1183/ 23120541.00147-2021].

\section{Abstract}

Cheyne-Stokes respiration (CSR) may trigger ventricular arrhythmia in patients with heart failure with reduced ejection fraction (HFrEF) and central sleep apnoea (CSA). This study determined the prevalence and predictors of a high nocturnal ventricular arrhythmia burden in patients with HFrEF and CSA (with and without CSR) and to evaluate the temporal association between CSR and the ventricular arrhythmia burden.

This cross-sectional ancillary analysis included 239 participants from the SERVE-HF major sub-study who had HFrEF and CSA, and nocturnal ECG from polysomnography. CSR was stratified in $\geqslant 20 \%$ and $<20 \%$ of total recording time (TRT). High burden of ventricular arrhythmia was defined as $>30$ premature ventricular complexes (PVCs) per hour of TRT. A sub-analysis was performed to evaluate the temporal association between CSR and ventricular arrhythmias in sleep stage N2.

High ventricular arrhythmia burden was observed in $44 \%$ of patients. In multivariate logistic regression analysis, male sex, lower systolic blood pressure, non-use of antiarrhythmic medication and CSR $\geqslant 20 \%$ were significantly associated with PVCs $>30 \cdot \mathrm{h}^{-1}$ (OR 5.49, 95\% CI 1.51-19.91, p=0.010; OR 0.98, 95\% CI 0.97-1.00, $\mathrm{p}=0.017$; OR 5.02, 95\% CI 1.51-19.91, $\mathrm{p}=0.001$; and OR 2.22, 95\% CI 1.22-4.05, $\mathrm{p}=0.009$; respectively). PVCs occurred more frequently during sleep phases with versus without CSR (median (interquartile range): $64.6(24.8-145.7)$ versus $34.6(4.8-75.2) \cdot \mathrm{h}^{-1} \mathrm{~N} 2$ sleep; $\left.\mathrm{p}=0.006\right)$.

Further mechanistic studies and arrhythmia analysis of major randomised trials evaluating the effect of treating CSR on ventricular arrhythmia burden and arrhythmia-related outcomes are warranted to understand how these data match with the results of the parent SERVE-HF study.

Introduction

Patients with heart failure are at increased risk of sudden cardiac death and ventricular arrhythmia [1]. In addition, a high burden of ventricular arrhythmia ( $>30$ premature ventricular complexes (PVCs) per hour) and a very high burden of ventricular arrhythmia (PVCs $>4 \%$ of total beats or $>10000$ PVCs/24 h) have 
been linked to the occurrence of higher grade ventricular arrhythmias (e.g. (non-)sustained ventricular tachycardias) [1], impaired left ventricular systolic function [2-4] and higher mortality [4-7].

Approximately $50 \%$ of patients with heart failure and reduced left ventricular ejection fraction (HFrEF) present with moderate to severe sleep-disordered breathing (SDB) [8]. Of those, about 50\% have predominant obstructive sleep apnoea (OSA), 50\% have predominant central sleep apnoea (CSA) and $41 \%$ show periods with a Cheyne-Stokes respiration (CSR) breathing pattern, irrespective of the type of SDB [9].

Pathophysiologically, heart failure can induce and worsen CSA via pulmonary congestion, hyperventilation and ventilatory control instability [10]. Additionally, CSA, and in particular CSR, may also contribute to the progression of heart failure [11]. Small studies showed that just a small proportion of patients with HFrEF and concomitant OSA have a high burden of ventricular arrhythmia [12, 13]. The association between CSA (and CSR) and PVCs in HFrEF populations remains unclear [12-15].

The aims of this study were to determine the prevalence of a high or a very high burden of nocturnal ventricular arrhythmia in patients with HFrEF and CSA (with and without CSR), to identify predictors and risk factors for such arrhythmia and, finally, to evaluate the temporal association between CSR and ventricular arrhythmia.

\section{Methods}

Study design

A cross-sectional ancillary analysis of data from participants with HFrEF and CSA in the SERVE-HF major sub-study (NCT00733343) was performed. The temporal association between CSR with ventricular arrhythmias in sleep stage N2 was performed in a subset of these patients.

\section{Study population}

Of 91 centres participating in SERVE-HF, seven contributed patients to the ancillary analysis of the major sub-study [16]. Participants in the sub-study were a subgroup of those enrolled in the SERVE-HF trial. Inclusion and exclusion criteria of the SERVE-HF major sub-study have been previously reported in detail $[11,16]$. Briefly, patients were aged $\geqslant 22$ years and had symptomatic chronic heart failure (New York Heart Association (NYHA) class III or IV, or class II with $\geqslant 1$ heart failure-related hospitalisation in the previous 24 months) and reduced left ventricular ejection fraction (LVEF $\leqslant 45 \%$ ) [16]. All received stable, contemporary guideline-based medical treatment for heart failure [16]. Use of antiarrhythmic drugs (chiefly amiodarone) was at the discretion of the investigators. With respect to SDB, individuals had predominant CSA [16]. Study exclusion criteria were amyloidosis, hypertrophic cardiomyopathy and diuretic dosage more than doubled within the 4 weeks prior to randomisation [16]. Additional exclusion criteria for this sub-analysis of the SERVE-HF major sub-study were insufficient ECG data (e.g. technical interference) and unclear rhythm or no consensus in expert round ( supplementary table S1).

The sub-study protocol was approved by the appropriate local or regional ethics committee [16]. The trial was conducted according to Good Clinical Practice and the Principles of the Declaration of Helsinki 2002. All participants gave written informed consent.

\section{CSA and Cheyne-Stokes respiration}

Sub-study evaluations such as demographics or polysomnography (PSG) were performed at the baseline visit [16]. CSA was defined as an apnoea-hypopnoea index (AHI) $>15 \cdot h^{-1}$ with $\geqslant 50 \%$ central events and a central AHI $\geqslant 10 \cdot \mathrm{h}^{-1}$, derived from PSG and based on total recording time (TRT), documented within 4 weeks of randomisation, with flow measurement performed using a nasal cannula [11]. All PSGs were centrally scored in a blinded fashion (HP2 Sleep CoreLab, Alpes University, Grenoble, France) by two scorers according to American Academy of Sleep Medicine (AASM) rules [17], with additional scoring by a third senior scorer if there were any discrepancies. CSR was defined as $\geqslant 3$ episodes of continuous cycles of waxing and waning tidal volumes with periods of hyperventilation separated by apnoea/hypopnoeas and visually quantified by the percentage of the recording time: $\geqslant 20 \%$ and $<20 \%$ (including those with no CSR) [11].

\section{ECG measurements - cardiac arrhythmias}

ECG data were derived from full overnight PSG. The nocturnal 1-lead-Holter ECG was monitored from a single precordial lead with sampling frequency of $250 \mathrm{~Hz}$. This frequency is acceptable for analysis of ventricular and supraventricular arrhythmias [18]. A single centre study with 167 patients suggested that PVC burden is similar in the morning (clock time 06:00-13:59), the afternoon (14:00-21:59) and at 
night-time (22:00-05:59) [19] in patients with HFrEF and SDB. Thus, nocturnal PVC burden assessed from nocturnal ECG is likely to provide a representative estimate of the total (24-h) PVC burden.

Data sets were visually analysed by two trained investigators (LG, JB) according to standard definitions [20], with low interobserver variability for PVCs comparable with previous important analyses of nocturnal ECGs in cohorts with PSG [18, 21] (intraclass correlation coefficients (95\% confidence interval) for a random sample of 20 sleep studies: 0.90 (0.75-0.96), $\mathrm{p}<0.001$; further details in the online supplementary material). Investigators were blinded with respect to clinical data and only had access to the ECG channel and the pre-scored PSG sleep stages. High and very high burden of ventricular arrhythmia was defined as PVCs $>30 \cdot \mathrm{h}^{-1}$ and $>4 \%$ PVCs of total beats or $>10000$ PVCs/24 h, respectively [2-7] (see online supplementary material for more details). In addition, a subset analysis $(n=19$, supplementary figure S1) was performed to analyse the occurrence of PVCs $\cdot \mathrm{h}^{-1}$ in sleep stage N2 in episodes with and without CSR $[15,22]$ (see online supplementary material for more details).

\section{Study outcomes}

The end-points in this analysis were the prevalence of high and very high burden of nocturnal ventricular arrhythmia at baseline, the identification of predictors of ventricular arrhythmia, and the temporal relationship between CSR and high ventricular arrhythmia burden.

\section{Statistical analysis}

Categorical data are presented as frequency in percentage and compared using the Chi-square test. Normally distributed quantitative data are expressed as mean \pm standard deviation, and non-normal data as median and interquartile range (IQR). Comparisons between quantitative variables were done with either unpaired t-test or Mann-Whitney U-test. Dependent, non-normally distributed, variables were compared using Wilcoxon signed-rank test. Univariate linear regression models were performed to assess the association between high ventricular arrhythmia burden and demographics/cardiac risk factors. A multivariate logistic regression model, including all independent variables with $p<0.1$ in the univariate model, was calculated. All p-values are two-sided, and p=0.05 was considered the threshold for statistical significance. Intraclass correlation (ICC) estimates and their 95\% confidence intervals were calculated based on a mean-rating $(k=2)$, absolute-agreement and 2-way mixed-effects model. Data entry and calculation were performed with the software package SPSS 26.0 (Chicago, IL, USA).

\section{Results}

\section{Patients}

Of the 312 participants in the SERVE-HF major sub-study, 239 had ECGs that fulfilled technical requirements (supplementary figure S1). Participants were primarily males (91\%), with a mean age of 69 years and an average ejection fraction of 33\% (table 1), similar to the parent SERVE-HF trial population [12]. The most common heart failure aetiology was ischaemic (57\%), and $60 \%$ had a cardiac implantable electronic device (table 1). Fourteen per cent of patients were documented as being on an antiarrhythmic drug (excluding $\beta$-blockers, which were documented separately), primarily amiodarone. The subgroup of patients with a high burden of ventricular arrhythmia (PVCs $>30 \cdot \mathrm{h}^{-1}$ ) were more likely to be male versus those with fewer ventricular arrhythmias (PVCs $\leqslant 30 \cdot \mathrm{h}^{-1}$ ); patients with PVCs $>30 \cdot \mathrm{h}^{-1}$ also had lower systolic blood pressure and were less likely to be using antiarrhythmics (table 1).

\section{Respiratory and sleep characteristics}

Study patients had a median AHI of $37 \cdot \mathrm{h}^{-1}$ with predominant CSA (cAHI/AHI 81\%). Severity and type of sleep apnoea were similar in patients with PVCs $>30 \cdot \mathrm{h}^{-1}$ or $\leqslant 30 \cdot \mathrm{h}^{-1}$ (supplementary table S2). Oxygen saturation, time with oxygen saturation $<90 \%$ and Epworth Sleepiness Scale scores were similar between groups. The proportion of sleep spent in slow-wave sleep or rapid eye movement sleep was low in both the PVCs $>30 \cdot \mathrm{h}^{-1}$ and $\leqslant 30 \cdot \mathrm{h}^{-1}$ groups (supplementary table S2).

\section{Prevalence of high burden of ventricular arrhythmia in patients with HFrEF and CSA}

The average number of PVCs was $25 \cdot \mathrm{h}^{-1}$. The proportion of patients with PVCs $>30 \cdot \mathrm{h}^{-1},>4 \%$ of total beats and $>10000 / 24$ h was $44 \%, 13 \%$ and $7 \%$, respectively. Nocturnal heart rate was similar in the groups with PVCs $>30 \cdot \mathrm{h}^{-1}$ and $\leqslant 30 \cdot \mathrm{h}^{-1}$ (table 2). In addition to the absolute number of PVCs/night, the proportions with $>4 \%$ PVCs/total beats and $>10000 \mathrm{PVCs} / 24 \mathrm{~h}$, and non-sustained ventricular tachycardia (NSVT), were higher in patients with PVCs $>30 \cdot \mathrm{h}^{-1}$ versus $\leqslant 30 \cdot \mathrm{h}^{-1}$ (table 2).

\section{Predictors and risk factors for high burden of ventricular arrhythmia}

In univariate analysis, male sex, low systolic blood pressure, haemoglobin, non-use of antiarrhythmics and CSR $\geqslant 20 \%$ were associated with PVCs $>30 \cdot \mathrm{h}^{-1}$ (table 3 ). All of these variables but haemoglobin 


\begin{tabular}{|c|c|c|c|c|}
\hline Baseline characteristics & Total & PVCs $\leqslant 30 \cdot h^{-1}$ & PVCs $>30 \cdot \mathrm{h}^{-1}$ & $\mathrm{p}$-value \\
\hline Subjects $n$ & 239 & 134 & 105 & \\
\hline Age years & $69.1 \pm 10.2$ & $69.7 \pm 10.5$ & $68.4 \pm 9.7$ & 0.310 \\
\hline Male n (\%) & $218(91 \%)$ & $116(87 \%)$ & $102(97 \%)$ & 0.004 \\
\hline Body mass index $\mathrm{kg} \cdot \mathrm{m}^{-2}$ & $29.4 \pm 5.1^{\#}$ & $29.1 \pm 4.6$ & $29.8 \pm 5.7$ & 0.285 \\
\hline Diabetes mellitus n (\%) & $109(46 \%)^{\#}$ & $60(45 \%)$ & $49(47 \%)$ & 0.619 \\
\hline NYHA class n (\%) & & & & 0.337 \\
\hline I or II & $58(24 \%)$ & $29(22 \%)$ & $29(27 \%)$ & \\
\hline III & $181(76 \%)$ & $105(78 \%)$ & $76(72 \%)$ & \\
\hline IV & $0(0 \%)$ & $0(0 \%)$ & $0(0 \%)$ & \\
\hline BNP $\mathrm{pg} \cdot \mathrm{mL}^{-1}$ & $2253.1 \pm 3085.1^{\natural,+}$ & $2119.1 \pm 2611.9$ & $2427.1 \pm 3619.4$ & 0.512 \\
\hline 6-min walk distance $\mathrm{m}$ & $339.7 \pm 124.8^{\S, f}$ & $331.6 \pm 121.5$ & $350.5 \pm 129.0$ & 0.264 \\
\hline $\operatorname{LVEF}^{\# \#} \%$ & $33.2 \pm 8.3$ & $33.8 \pm 7.7$ & $32.3 \pm 8.9$ & 0.163 \\
\hline Heart failure aetiology $n(\%)$ & & & & 0.362 \\
\hline Ischaemic & $141(59 \%)$ & $77(58 \%)$ & $64(61 \%)$ & \\
\hline Other & $98(41 \%)$ & $57(42 \%)$ & $41(39 \%)$ & \\
\hline \multicolumn{5}{|l|}{ Blood pressure $\mathrm{mmHg}$} \\
\hline Systolic & $123.1 \pm 18.5^{\#, \text { ฯฯ }}$ & $125.3 \pm 20.1$ & $120.2 \pm 15.9$ & 0.032 \\
\hline Diastolic & $73.7 \pm 11.4^{\#, \text {,ฯฯ }}$ & $74.5 \pm 11.9$ & $72.6 \pm 10.7$ & 0.219 \\
\hline Implanted device $\mathrm{n}(\%)$ & & & & 0.756 \\
\hline None & $106(44 \%)$ & $58(43 \%)$ & $48(46 \%)$ & \\
\hline Non-CRT pacemaker & $12(5 \%)$ & $8(6 \%)$ & $4(3 \%)$ & \\
\hline$I C D$ & $66(28 \%)$ & $38(28 \%)$ & $28(27 \%)$ & \\
\hline CRT-P & $2(1 \%)$ & $1(1 \%)$ & $1(1 \%)$ & \\
\hline CRT-D & $53(22 \%)$ & $29(22 \%)$ & $24(23 \%)$ & \\
\hline Rhythm n (\%) & & & & 0.208 \\
\hline Sinus rhythm & $133(56 \%)^{\#}$ & $72(54 \%)$ & $61(59 \%)$ & \\
\hline Atrial fibrillation & $61(26 \%)$ & $32(24 \%)$ & $29(28 \%)$ & \\
\hline Other & $44(18 \%)$ & $30(22 \%)$ & $14(13 \%)$ & \\
\hline Diurnal heart rate/min & $70.0 \pm 12.1^{\#}$ & $69.3 \pm 12.4$ & $71.0 \pm 11.7$ & 0.308 \\
\hline Diurnal QRS duration ms & $133.4 \pm 37.3^{++}$ & $135.3 \pm 39.6$ & $130.9 \pm 34.1$ & 0.360 \\
\hline Diurnal QRS >120 ms & $125(53 \%)^{++}$ & $73(55 \%)$ & $52(51 \%)$ & 0.542 \\
\hline Bundle branch block $^{\#} \mathrm{n}(\%)$ & & & & 0.430 \\
\hline Right & $17(7 \%)$ & $9(7 \%)$ & $8(8 \%)$ & \\
\hline Left & $59(25 \%)$ & $38(28 \%)$ & $21(20 \%)$ & \\
\hline Other & $51(21 \%)$ & $25(19 \%)$ & $26(25 \%)$ & \\
\hline \multicolumn{5}{|l|}{ Cardiac medication $\mathrm{n}(\%)$} \\
\hline ACEI or ARB & $224(94 \%)$ & $128(96 \%)$ & $96(91 \%)$ & 0.195 \\
\hline$\beta$-blocker & $217(91 \%)$ & $121(90 \%)$ & $96(91 \%)$ & 0.764 \\
\hline Aldosterone antagonist & $142(59 \%)$ & $84(63 \%)$ & $58(55 \%)$ & 0.244 \\
\hline Diuretic & $201(84 \%)$ & $110(82 \%)$ & $91(87 \%)$ & 0.337 \\
\hline Cardiac glycoside & $51(21 \%)$ & $31(23 \%)$ & $20(19 \%)$ & 0.444 \\
\hline Antiarrhythmics & $33(14 \%)$ & $27(20 \%)$ & $6(6 \%)$ & 0.001 \\
\hline Creatinine ${ }^{\S \S} \mathrm{mg} \cdot \mathrm{dL}^{-1}$ & 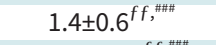 & $1.4 \pm 0.7$ & $1.4 \pm 0.5$ & 0.591 \\
\hline eGFR $\mathrm{mL} \cdot \mathrm{min}^{-1} \cdot 1.73 \mathrm{~m}^{-2}$ & $58.8 \pm 21.6^{f f, \ldots \# \#}$ & $58.3 \pm 22.3$ & $59.5 \pm 20.6$ & 0.671 \\
\hline Haemoglobin $\mathrm{g} \cdot \mathrm{dL}^{-1}$ & $14.0 \pm 1.5^{થ ฯ ฯ ฯ,+++}$ & $13.8 \pm 1.5$ & $14.3 \pm 1.5$ & 0.025 \\
\hline \multicolumn{5}{|c|}{ 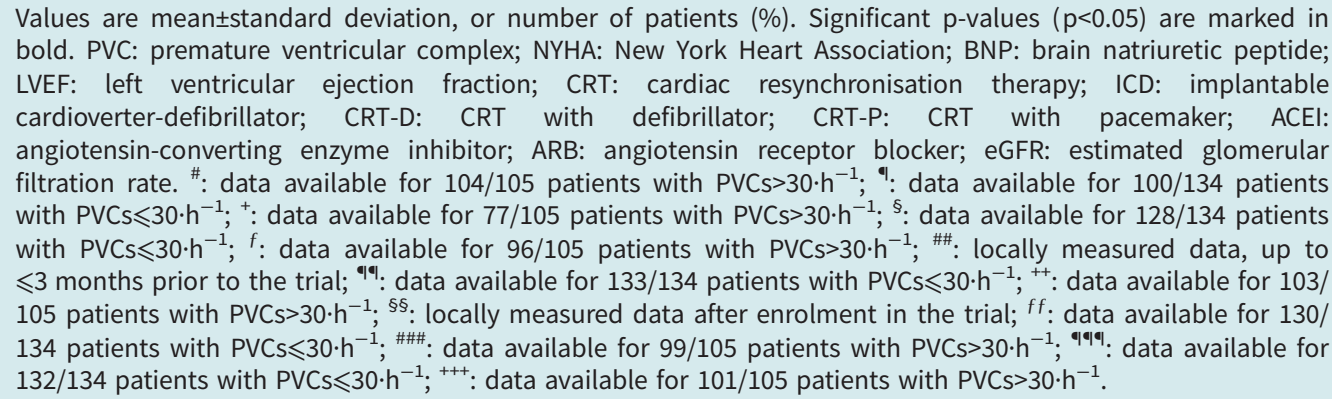 } \\
\hline
\end{tabular}




\begin{tabular}{|c|c|c|c|c|}
\hline & Total & PVCs $\leqslant 30 \cdot h^{-1}$ & PVCs $>30 \cdot h^{-1}$ & p-value \\
\hline Subjects n & 239 & 134 & 105 & \\
\hline ECG recording time $h$ & $7.4(6.7-8.0)$ & $7.5(6.8-8.1)$ & $7.4(6.6-7.9)$ & 0.268 \\
\hline Nocturnal heart rate beats/min & $67(60-74)$ & $65(59-74)$ & $69(63-75)$ & 0.047 \\
\hline \multicolumn{5}{|l|}{ Ventricular arrhythmias/h TRT } \\
\hline PVCs & $25(2.5-77.4)$ & $3.3(0.8-13.0)$ & $85.2(48.6-241.7)$ & $<0.001$ \\
\hline Ventricular couplets & $0.3(0.0-2.4)$ & $0.0(0.0-0.1)$ & $3.1(0.7-7.5)$ & $<0.001$ \\
\hline \multicolumn{5}{|c|}{ Higher grade ventricular arrhythmias $n(\%)$} \\
\hline$>4 \%$ PVCs of TRT & $31(13 \%)$ & $0(0 \%)$ & $31(30 \%)$ & $<0.001$ \\
\hline >10000 PVCs/24 h & $17(7 \%)$ & $0(0 \%)$ & $17(16 \%)$ & $<0.001$ \\
\hline$\geqslant 1$ episode of non-sustained VT & $50(21 \%)$ & $21(16 \%)$ & $29(28 \%)$ & 0.024 \\
\hline
\end{tabular}

Values are median (interquartile range), or number of patients $(\%)$. Significant $p$-values $(p<0.05)$ are marked in bold. PVC: premature ventricular complex; TRT: total recording time; VT: ventricular tachycardia.

remained significantly associated with PVCs $>30 \cdot \mathrm{h}^{-1}$ in the adjusted multivariate analysis (table 3). Patients with CSR $\geqslant 20 \%$ had significantly more PVCs/h than those with a CSR proportion $<20 \%$ group (figure $1 \mathrm{a}$ ), and PVCs $>30 \cdot \mathrm{h}^{-1}$ was more prevalent in the $\mathrm{CSR} \geqslant 20 \%$ group (figure $1 \mathrm{~b}$ ).

High burden of ventricular arrhythmia in sleep phases with CSR

A subset of patients ( $\mathrm{n}=19$; supplementary figure S1) was eligible for analysis of PVCs/h during sleep stage N2 in phases with versus without CSR [15, 22]. Baseline data for this subset are presented in supplementary table S3. In total, $54.5 \mathrm{~h}$ of sleep stage N2 were analysed. The cumulative length of episodes with and without CSR were 26.1 and $28.4 \mathrm{~h}$, respectively. PVCs/h occurred more frequently during episodes with versus without CSR (figure 2). Figure 3 represents a typical PSG recording showing the occurrence of PVCs in phases of no CSR versus CSR.

TABLE 3 Univariate and multivariate logistic regression models analysing the association of preselected variables with premature ventricular complexes $>30 \cdot \mathrm{h}^{-1}$

\begin{tabular}{|c|c|c|c|c|c|}
\hline & \multirow[t]{2}{*}{ n } & \multicolumn{2}{|c|}{ Univariate analysis } & \multicolumn{2}{|c|}{ Multivariate analysis" } \\
\hline & & OR $(95 \% \mathrm{Cl})$ & p-value & OR $(95 \% \mathrm{CI})$ & p-value \\
\hline Age years & 239 & $0.99(0.96-1.01)$ & 0.309 & & \\
\hline Male sex & 239 & $5.28(1.51-18.43)$ & 0.009 & $4.63(1.27-16.97)$ & 0.021 \\
\hline Body mass index $\mathrm{kg} \cdot \mathrm{m}^{-2}$ & 238 & $1.03(0.98-1.08)$ & 0.285 & & \\
\hline Systolic BP mmHg & 237 & $0.99(0.97-1.00)$ & 0.039 & $0.98(0.96-1.00)$ & 0.010 \\
\hline Diastolic BP $\mathrm{mmHg}$ & 237 & $0.99(0.96-1.01)$ & 0.218 & & \\
\hline Heart rate beats $\cdot \min ^{-1}$ & 238 & $1.01(0.99-1.03)$ & 0.307 & & \\
\hline Atrial fibrillation & 238 & $0.81(0.45-1.46)$ & 0.483 & & \\
\hline LVEF \% & 239 & $0.98(0.95-1.01)$ & 0.163 & & \\
\hline Plasma BNP pg.mL $\mathrm{mL}^{-1}$ & 177 & $1.00(1.00-1.00)$ & 0.512 & & \\
\hline QRS duration ms & 237 & $1.00(0.99-1.00)$ & 0.368 & & \\
\hline Serum creatinine $\mathrm{mg} \cdot \mathrm{dL}^{-1}$ & 229 & $0.88(0.56-1.39)$ & 0.591 & & \\
\hline Haemoglobin $\mathrm{g} \cdot \mathrm{dL}^{-1}$ & 188 & $1.22(1.02 ; 1.46)$ & 0.026 & $1.20(0.99 ; 1.46)$ & 0.062 \\
\hline Non-use of antiarrhythmics & 239 & $4.16(1.65-10.51)$ & 0.003 & $4.43(1.68-11.65)$ & 0.003 \\
\hline $\boldsymbol{\beta}$-blocker & 239 & $1.15(0.47-2.79)$ & 0.764 & & \\
\hline Cardiac glycoside & 239 & $0.78(0.42-1.47)$ & 0.445 & & \\
\hline Apnoea-hypopnoea index/h & 239 & $0.99(0.97-1.01)$ & 0.193 & & \\
\hline CSR $\geqslant 20 \%$ & 238 & 2.09 (1.19-3.69) & 0.011 & $2.38(1.28-4.45)$ & 0.006 \\
\hline Time with oxygen saturation $<90 \%, \%$ & 239 & $0.99(0.98-1.00)$ & 0.158 & & \\
\hline
\end{tabular}



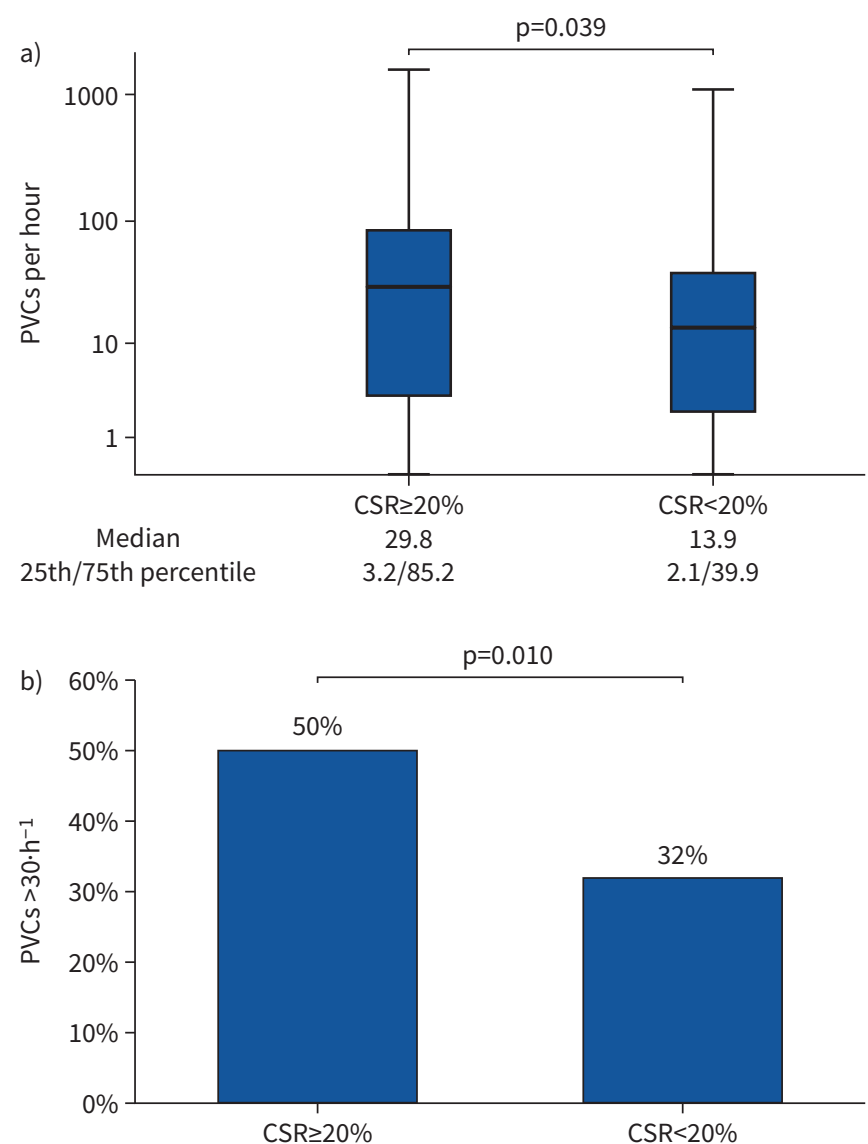

FIGURE 1 a) Boxplot showing premature ventricular complexes (PVCs) per hour in patients with Cheyne-Stokes respiration (CSR) $\geqslant 20 \%(n=161)$ versus $<20 \%(n=78)$. Data are expressed as median, minimum, maximum, 25 th percentile and 75th percentile on a logarithmic scale. b) Prevalence of frequent premature ventricular complexes $\left(>30 \mathrm{~h}^{-1}\right)$ in patients with $\mathrm{CSR} \geqslant 20 \%$ versus $<20 \%$. Data are expressed as percentage.

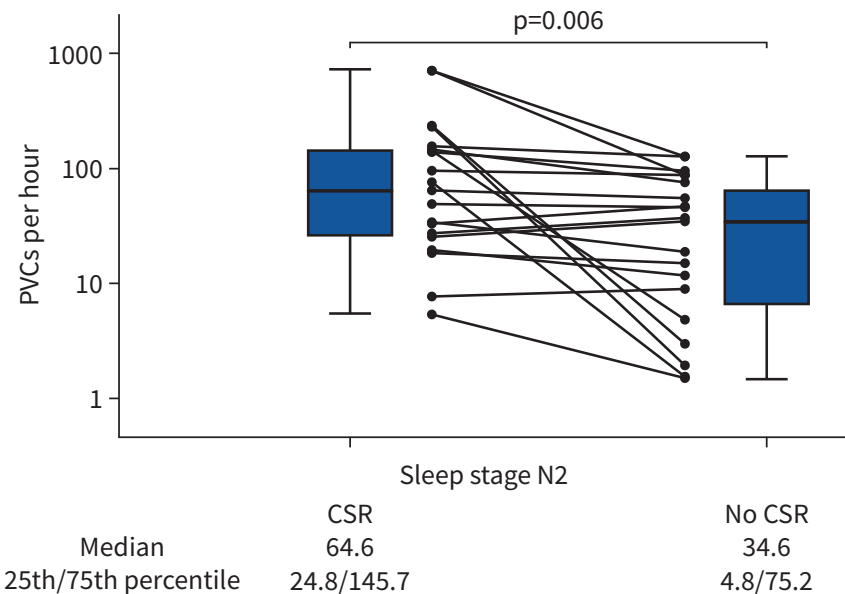

FIGURE 2 Boxplot showing premature ventricular complexes per hour in sleep stage N2 in episodes with $(26.1 \mathrm{~h})$ versus without Cheyne-Stokes respiration (CSR, 28.4 h). Data are expressed as median, minimum, maximum, 25th percentile and 75th percentile on a logarithmic scale. Individual data on premature ventricular complexes (PVCs) for the 19 included patients are also shown, 15 of whom showed a higher number of PVCS per hour in periods with versus without CSR. 


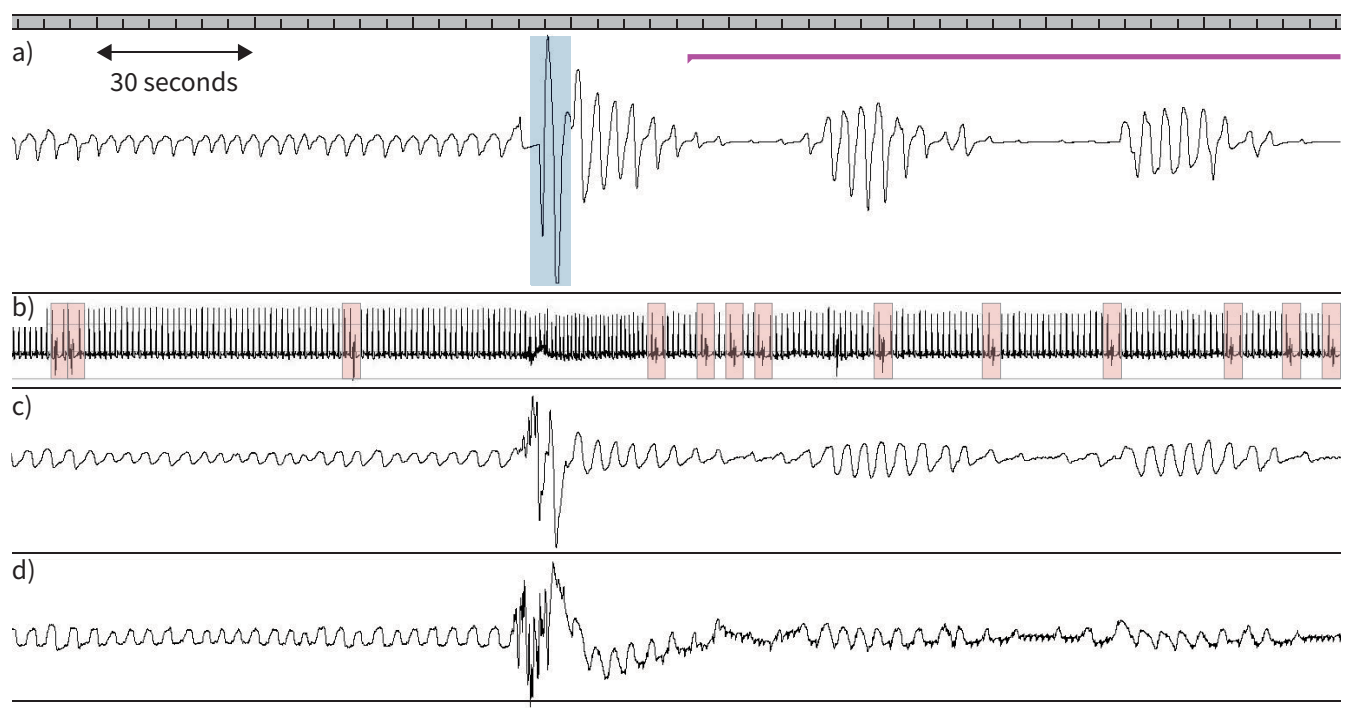

Amplitude of tidal volume at least twice as high compared to normal ventilation

Cheyne-Stokes respiration

Premature ventricular complex

FIGURE 3 Comparison of premature ventricular complexes (PVCs) in phases with and without Cheyne-Stokes respiration (CSR) during sleep (representative polysomnographic recording). The ratio of PVC in phases with and without CSR was 2:1. a) Nasal airflow: typical triggering of CSR in patients with heart failure with reduced ejection fraction by relative hyperventilation [40] marked in blue. The pink line represents a phase of CSR. b) Electrocardiogram (PVCs marked in orange). c) Effort thorax. d) Effort abdomen.

\section{Discussion}

This study provides novel insights into the association between high burden of ventricular arrhythmia and CSA in patients with HFrEF. The prevalence of high burden of ventricular arrhythmia (PVCs $>30 \cdot \mathrm{h}^{-1}$ ) in patients with HFrEF and CSA was 44\%. Factors associated with higher rates of PVCs $>30 \cdot \mathrm{h}^{-1}$ in multivariate logistic regression analysis were male sex, low systolic blood pressure, non-use of antiarrhythmics and CSR $\geqslant 20 \%$. In addition, high rates of PVC were significantly more frequent in sleep phases with CSR.

Despite the fact that ventricular arrhythmias are common in up to $97 \%$ of patients with HFrEF [23], and that CSA and CSR may trigger ventricular arrhythmias due to their inherent pathophysiology $[15,18]$, data on the burden of ventricular arrhythmias in HFrEF patients with CSA from larger studies are scarce. LANFRANCHI et al. [14] studied patients with LVEF $\leqslant 40 \%$ and $\mathrm{AHI} \geqslant 15 \cdot \mathrm{h}^{-1}$. In 17 patients with severe CSA (AHI $43 \cdot \mathrm{h}^{-1}$ ) and LVEF $27 \%$ the rate of nocturnal PVCs was $95 \cdot \mathrm{h}^{-1}$. In contrast, data from another study in comparable patients (CSA with AHI $41 \cdot \mathrm{h}^{-1}$ and LVEF 30\%) reported a lower rate of PVCs $\left(34 \cdot h^{-1}\right)$ [19]. Both studies used 24-h Holter ECG for analysis [14, 19]. In contrast, in the current multicentre analysis, ECG and respiratory data were recorded the same night and an even lower PVC burden was recorded $\left(25 \cdot h^{-1}\right)$. However, nearly half of all patients had a rate of PVCs $\geqslant 30 \cdot h^{-1}$, while $13 \%$ had PVCs $>4 \%$ of total beats and $7 \%$ showed $>10000$ PVCs/24 h. This finding is noteworthy because the risk of sudden cardiac death in the general population with high or very high burden of ventricular arrhythmia is increased by 2.6-fold versus those with a lower ventricular arrhythmia burden [4-7].

Comparable studies in this distinctive HFrEF population with CSA and high or very high ventricular arrhythmia burden have not been performed yet. Studies evaluating different cohorts such as SDB patients undergoing cardiac surgery [24] or without cardiac disease [25] reported much lower rates of high ventricular arrhythmia burden of $19 \%$ and $3 \%$, respectively.

Factors such as male sex, low blood pressure, non-use of antiarrhythmics and CSR $\geqslant 20 \%$ were significantly associated with high burden of ventricular arrhythmias in patients with HFrEF and CSA in our study. Other known risk factors such as age and ejection fraction were not associated with high burden of ventricular arrhythmias [1], possibly due to the pre-selection of patients with HFrEF. Similar to our results, others reported strong associations between male sex [26], low systolic blood pressure [27] and non-use of antiarrhythmics [1] and a high burden of ventricular arrhythmias. 
We found that CSR $\geqslant 20 \%$ was associated with a high burden of ventricular arrhythmia. CSR $\geqslant 20 \%$ remained an independent risk factor after adjusting for potential confounders such as sex, systolic blood pressure and use of antiarrhythmic drugs.

When analysing episodes with and without CSR using 19 patients as their own controls [15, 22], and therefore eliminating potential confounders, we found that the burden of PVCs/h was $86 \%$ higher during periods of CSR. LEUng et al. [15] has previously reported a doubling of PVC burden in nine patients with HFrEF and CSA in phases with versus without CSR. The absolute burden of PVCs/h in Leung et al. [15] was two-fold higher than in our study. Four of the nine patients had no analysable phases without CSR. In these patients, regular breathing was artificially induced by $\mathrm{CO}_{2}$ inhalation, which was associated with a significant reduction in PVCs [15].

Key mechanisms of how CSA may induce a high burden of ventricular arrhythmia in HFrEF include activation of the sympathetic nervous system, left ventricular wall stress and hypoxia [28]. In addition, the large tidal volumes generated during the hyperpnoeic phase of CSR-CSA are indicative of intense respiratory drive [15]. Since activation of brainstem respiratory neurons can coactivate adjacent central sympathetic neurons in animal preparations [15, 29], surges in respiratory drive during the hyperpnoeic phase of CSR-CSA in humans could stimulate phase-linked bursts of central sympathetic outflow [15].

Moreover, CSRs induce mechanical myocardial distension [30] and may increase heart rate [31].

Long-term, exposure to CSA and CSR can contribute to chronic structural ventricular remodelling processes, increasing PVC susceptibility; furthermore, acutely, transient changes in ventricular electrophysiology and haemodynamics may directly trigger PVCs during specific CSA episodes [32].

However, the evidence for arrhythmogenic mechanisms related to CSA and CSR is not completely clear. In a pig model of sleep apnoea, mainly simulated obstructive, but not central apnoeas, resulted in transient changes in ventricular repolarisation, potentially increasing arrhythmia risk [33]. Additionally, it has been proposed that CSR may be, at least partially, protective in patients with HFrEF [34]. Potential protective mechanism of CSR includes increased end-expiratory lung volume followed by better oxygenation, respiratory alkalosis and hypocapnia, leading to favourable effects on heart muscle viability during hypoxia and better myocardial oxygen delivery [34]. Moreover, in a mathematical model, periodic muscular load followed by recovery, resembling CSR, has been shown to be favourable compared with continuous work to compensate for pre-existing hypoxia [35].

Irrespective of CSR, a high burden of ventricular arrhythmia has been reported to be associated with higher grade ventricular arrhythmias and mortality [4, 6, 7]. In HFrEF patients, those with CSR have been reported to have higher mortality compared to those without CSR [36, 37], and there is a dose-response relationship between CSR and mortality rate [37]. The observed association between CSR and high ventricular arrhythmia burden as well as the temporal relationship between sleep phases with CSR and increased PVC burden suggests, but does not prove, a causal relationship between CSR and ventricular arrhythmias, and a may be considered a potential mechanism for increased mortality in HFrEF patients with CSR.

Despite this, abolition of CSA with adaptive servo-ventilation (ASV) therapy in HFrEF patients in the parent randomised trial (SERVE-HF) was associated with a 34\% increase in cardiovascular mortality [11], largely driven by an increase in deaths without a preceding hospitalisation and therefore most likely sudden cardiac deaths [38]. Interestingly, a post hoc analysis of the SERVE-HF study showed that patients with $\mathrm{CSR} \geqslant 20 \%$ were more likely to experience a primary end-point event (all-cause death or life-saving cardiovascular intervention plus unplanned hospitalisation for worsening chronic heart failure) when randomised to ASV versus the control group, while the risk of an end-point event in those with CSR $<20 \%$ was lower in the ASV group versus control [11].

Thus, our finding of an association between CSR and ventricular arrhythmia burden in HFrEF patients with CSA could be interpreted that HFrEF with CSR have a higher susceptibility for ventricular arrhythmias, without direct causality. Alternatively, CSR may contribute to trigger ventricular arrhythmias, which has to be confirmed in mechanistic clinical studies or interventional trials with longer simultaneous rhythm and sleep apnoea monitoring.

The results from this sub-analysis must be interpreted in the light of several limitations. A direct causal relationship cannot be inferred due to the cross-sectional study design. In this study, only nocturnal PVC 
burden could be analysed. A 24-h Holter ECG was not available, and we did not have systematic collection of data from the cardiac implantable electronic devices in the parent or this sub-study. The association between CSR and rare higher grade ventricular arrhythmias such as NSVT could not be adequately addressed due to limited statistical power. However, consistent with existing literature, data indicate that a high burden of ventricular arrhythmia is associated with higher grade ventricular arrhythmias [1]. Thus, high ventricular arrhythmia burden should be a legitimate surrogate for higher grade ventricular arrhythmias. Specific antiarrhythmic drugs were not assessed in this analysis, although the majority were taking amiodarone in our population [38]. Intrathoracic pressures, tidal volumes and markers of autonomic dysfunction were not assessed. Sleeping position, which may have an effect on the number and nature (obstructive/central) of apnoeas and hypopnoeas, was not systematically assessed.

A strength of the current study is that PSG and ECG data were obtained on the same night, in contrast to other publications $[14,19,24]$. Therefore, possible confounders such as time-related effects should be minimal.

In conclusion, $44 \%$ of patients with HFrEF and CSA had a high burden of ventricular arrhythmia, and this was more likely in males, those with CSR $\geqslant 20 \%$ of the recording time, when systolic blood pressure was lower and in the absence of antiarrhythmic medication. Ventricular arrhythmia burden was higher during sleep with CSR than without CSR. Further mechanistic studies and arrhythmia analysis of major randomised trials (e.g. SERVE-HF [11] or ADVENT-HF [39]) evaluating the effect of treating CSR on ventricular arrhythmia burden and arrhythmia-related outcomes are warranted to understand how these data match with the results of the parent SERVE-HF study [11].

Acknowledgements: Medical writing assistance was provided by Nicola Ryan, independent medical writer, funded by ResMed. The authors thank the CRI (Clinical Research Institute) for their expertise in overseeing the SERVE-HF trial.

Author contributions: C. Fisser and M. Arzt were responsible for conceiving and designing the study and its hypotheses, acquiring study funding, collecting, analysing and interpreting the data, and writing and revising the manuscript prior to submission. C. Fisser, J. Bureck and L. Gall were involved in the collection, analysis and interpretation of data, and were responsible for drafting and revising the manuscript prior to submission. V. Vaas, J. Priefert, S. Fredersdorf, F. Zeman, D. Linz, H. Woehrle, R. Tamisier, M.R. Cowie and H. Teschler were involved in the collection and interpretation of data and critical revision of the manuscript prior to submission. F. Zeman was involved in data analysis and critical revision of the manuscript prior to submission.

Conflict of interest: C. Fisser reports grants from the German Heart Foundation/German Foundation of Heart Research during the conduct of the study. J. Bureck has nothing to disclose. L. Gall has nothing to disclose. V. Vaas reports grants from the German Society of Sleep Medicine outside the submitted work. J. Priefert has nothing to disclose. S. Fredersdorf has nothing to disclose. F. Zeman has nothing to disclose. D. Linz reports personal fees from Bayer, LivaNova, ResMed and Respicardia outside the submitted work. H. Wöhrle is a former employee of ResMed. R. Tamisier reports grants from ResMed and personal fees from Agiradom, outside the submitted work. H. Teschler reports grants and personal fees from ResMed outside the submitted work. M.R. Cowie reports grants and personal fees from ResMed, personal fees from Respicardia, and grants from Bayer, outside the submitted work. M. Arzt reports grants from ResMed during the conduct of the study; and personal fees from ResMed, grants and personal fees from Philips Respironics, personal fees from Boehringer Ingelheim, NRI, Novartis, JAZZ Pharmaceuticals, Inspire and Bresotec, and grants from the Else-Kroehner Fresenius Foundation, outside the submitted work.

Support statement: The SERVE-HF study was supported by ResMed and by grants from the National Institute for Health Research (NIHR) Cardiovascular and Respiratory Biomedical Research Units (MRC). Representatives and scientists from the ResMed participated in the study including design, data collation, data analysis and critical review of the paper. This ancillary analysis was supported by ResMed (M. Arzt) and by a grant from the German Heart Foundation/German Foundation of Heart Research (F/15/20; C. Fisser). Funding information for this article has been deposited with the Crossref Funder Registry.

\section{References}

1 Al-Khatib SM, Stevenson WG, Ackerman MJ, et al. 2017 AHA/ACC/HRS Guideline for Management of Patients with Ventricular Arrhythmias and the Prevention of Sudden Cardiac Death: A Report of the American College of Cardiology/American Heart Association Task Force on Clinical Practice Guidelines and the Heart Rhythm Society. J Am Coll Cardiol 2018; 72: e91-e220. 
2 Penela D, Acosta J, Aguinaga L, et al. Ablation of frequent PVC in patients meeting criteria for primary prevention ICD implant: safety of withholding the implant. Heart Rhythm 2015; 12: 2434-2442.

3 Kanei $\mathrm{Y}$, Friedman M, Ogawa N, et al. Frequent premature ventricular complexes originating from the right ventricular outflow tract are associated with left ventricular dysfunction. Ann Noninvasive Electrocardiol 2008; 13: 81-85.

4 Ataklte F, Erqou S, Laukkanen J, et al. Meta-analysis of ventricular premature complexes and their relation to cardiac mortality in general populations. Am J Cardiol 2013; 112: 1263-1270.

5 Berruezo A, Penela D, Jáuregui B, et al. Mortality and morbidity reduction after frequent premature ventricular complexes ablation in patients with left ventricular systolic dysfunction. Europace 2019; 21: 1079-1087.

6 Sajadieh A, Nielsen OW, Rasmussen V, et al. Ventricular arrhythmias and risk of death and acute myocardial infarction in apparently healthy subjects of age >or=55 years. Am J Cardiol 2006; 97: 1351-1357.

7 Bikkina M, Larson MG, Levy D. Prognostic implications of asymptomatic ventricular arrhythmias: the Framingham Heart Study. Ann Intern Med 1992; 117: 990-996.

8 Arzt $\mathrm{M}$, Woehrle $\mathrm{H}$, Oldenburg $\mathrm{O}$, et al. Prevalence and predictors of sleep-disordered breathing in patients with stable chronic heart failure: the SchlaHF registry. JACC Heart Fail 2016; 4: 116-125.

9 Arzt M, Oldenburg O, Graml A, et al. Phenotyping of sleep-disordered breathing in patients with chronic heart failure with reduced ejection fraction: the SchlaHF registry. J Am Heart Assoc 2017; 6: e005899.

10 Bradley TD, Floras JS. Sleep apnea and heart failure, Part II: central sleep apnea. Circulation 2003; 107: $1822-1826$.

11 Cowie MR, Woehrle $\mathrm{H}$, Wegscheider $\mathrm{K}$, et al. Adaptive servo-ventilation for central sleep apnea in systolic heart failure. N Engl J Med 2015; 373: 1095-1105.

12 Javaheri S, Parker TJ, Liming JD, et al. Sleep apnea in 81 ambulatory male patients with stable heart failure. Types and their prevalences, consequences, and presentations. Circulation 1998; 97: 2154-2159.

13 Ryan CM, Usui K, Floras JS, et al. Effect of continuous positive airway pressure on ventricular ectopy in heart failure patients with obstructive sleep apnoea. Thorax 2005; 60: 781-785.

14 Lanfranchi PA, Somers VK, Braghiroli A, et al. Central sleep apnea in left ventricular dysfunction: prevalence and implications for arrhythmic risk. Circulation 2003; 107: 727-732.

15 Leung RST, Diep TM, Bowman ME, et al. Provocation of ventricular ectopy by Cheyne-Stokes respiration in patients with heart failure. Sleep 2004; 27: 1337-1343.

16 Cowie MR, Woehrle $\mathrm{H}$, Wegscheider $\mathrm{K}$, et al. Adaptive servo-ventilation for central sleep apnoea in systolic heart failure: results of the major substudy of SERVE-HF. Eur J Heart Fail 2018; 20: 536-544.

17 Berry RB, Budhiraja R, Gottlieb DJ, et al. Rules for scoring respiratory events in sleep: update of the 2007 AASM Manual for the Scoring of Sleep and Associated Events. Deliberations of the Sleep Apnea Definitions Task Force of the American Academy of Sleep Medicine. J Clin Sleep Med 2012; 8: 597-619.

18 Mehra R, Stone KL, Varosy PD, et al. Nocturnal arrhythmias across a spectrum of obstructive and central sleep-disordered breathing in older men: outcomes of sleep disorders in older men (MrOS sleep) study. Arch Intern Med 2009; 169: 1147-1155.

19 Omran H, Bitter T, Horstkotte D, et al. Characteristics and circadian distribution of cardiac arrhythmias in patients with heart failure and sleep-disordered breathing. Clin Res Cardiol 2018; 107: 965-974.

20 O'Keefe JH, Hammill SC, Freed MS. Complete Guide to ECGs: A Comprehensive Study Guide to Improve ECG Interpretation Skills. 4th Edn. Sudbury, Jones \& Bartlett Learning, 2017.

21 Monahan K, Storfer-Isser A, Mehra R, et al. Triggering of nocturnal arrhythmias by sleep-disordered breathing events. J Am Coll Cardiol 2009; 54: 1797-1804.

22 Ryan CM, Juvet S, Leung R, et al. Timing of nocturnal ventricular ectopy in heart failure patients with sleep apnea. Chest 2008; 133: 934-940.

23 Szabó BM, van Veldhuisen DJ, Crijns HJ, et al. Value of ambulatory electrocardiographic monitoring to identify increased risk of sudden death in patients with left ventricular dysfunction and heart failure. Eur Heart J 1994; 15: 928-933.

24 Unosawa S, Sezai A, Akahoshi T, et al. Arrhythmia and sleep-disordered breathing in patients undergoing cardiac surgery. J Cardiol 2012; 60: 61-65.

25 Flemons WW, Remmers JE, Gillis AM. Sleep apnea and cardiac arrhythmias. Is there a relationship? Am Rev Respir Dis 1993; 148: 618-621.

26 Kannel WB, Wilson PW, D’Agostino RB, et al. Sudden coronary death in women. Am Heart J 1998; 136: 205-212.

27 Arundel C, Lam PH, Gill GS, et al. Systolic blood pressure and outcomes in patients with heart failure with reduced ejection fraction. J Am Coll Cardiol 2019; 73: 3054-3063.

28 Fisser C, Götz K, Hetzenecker A, et al. Obstructive sleep apnoea but not central sleep apnoea is associated with left ventricular remodelling after acute myocardial infarction. Clin Res Cardiol 2020; 39: 119.

29 Guyenet PG, Koshiya N, Huangfu D, et al. Central respiratory control of A5 and A6 pontine noradrenergic neurons. Am J Physiol 1993; 264: R1035-R1044. 

determinants of stretch-activated arrhythmias. Circulation 1992; 86: 968-978.

31 Winkle RA. The relationship between ventricular ectopic beat frequency and heart rate. Circulation 1982; 66: 439-446.

32 Linz D, McEvoy RD, Cowie MR, et al. Associations of obstructive sleep apnea with atrial fibrillation and continuous positive airway pressure treatment: a review. JAMA Cardiol 2018; 3: 532-540.

33 Linz D, Denner A, Illing S, et al. Impact of obstructive and central apneas on ventricular repolarisation: lessons learned from studies in man and pigs. Clin Res Cardiol 2016; 105: 639-647.

34 Linz D, Fox H, Bitter T, et al. Impact of SERVE-HF on management of sleep disordered breathing in heart failure: a call for further studies. Clin Res Cardiol 2016; 105: 563-570.

35 Levine M, Cleave JP, Dodds C. Can periodic breathing have advantages for oxygenation? J Theor Biol 1995; 172: 355-368.

36 Hanly PJ, Zuberi-Khokhar NS. Increased mortality associated with Cheyne-Stokes respiration in patients with congestive heart failure. Am J Respir Crit Care Med 1996; 153: 272-276.

37 Ancoli-Israel S, Engler RL, Friedman PJ, et al. Comparison of patients with central sleep apnea. With and without Cheyne-Stokes respiration. Chest 1994; 106: 780-786.

38 Eulenburg $\mathrm{C}$, Wegscheider $\mathrm{K}$, Woehrle $\mathrm{H}$, et al. Mechanisms underlying increased mortality risk in patients with heart failure and reduced ejection fraction randomly assigned to adaptive servoventilation in the SERVE-HF study: results of a secondary multistate modelling analysis. Lancet Respir Med 2016; 4: 873-881.

39 Lyons OD, Floras JS, Logan AG, et al. Design of the effect of adaptive servo-ventilation on survival and cardiovascular hospital admissions in patients with heart failure and sleep apnoea, The ADVENT-HF trial. Eur J Heart Fail 2017; 19: 579-587.

40 Naughton M, Benard D, Tam A, et al. Role of hyperventilation in the pathogenesis of central sleep apneas in patients with congestive heart failure. Am Rev Respir Dis 1993; 148: 330-338. 